ARTICLE

\title{
Approach to lower back pain
}

\author{
F Moosajee, MB ChB, FCP (SA); A A Kalla, MB ChB, FCP (SA), MD \\ Division of Rheumatology, Department of Medicine, Faculty of Health Sciences, University of Cape Town and Groote Schuur Hospital, \\ Cape Town, South Africa
}

Corresponding author: F Moosajee(farzana_moosajee@yahoo.com)

Lower back pain is one of the most common symptoms - and the most common musculoskeletal problem - seen by general practitioners. It is also a common cause of disability and an expensive condition in terms of economic impact because of absenteeism. This article discusses an approach to this common symptom and how to distinguish the benign, mechanical type of back pain from the more sinister, but less frequently encountered, inflammatory back pain.

S Afr Med J 2015;105(12):1077. DOI:10.7196/SAMJ.2015.v105i12.10249

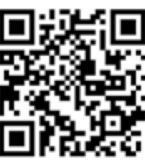

Lower back pain (LBP) is the most common musculoskeletal problem seen by general practitioners and remains the most common cause of disability in US patients $<45$ years of age. ${ }^{[1-3]}$ It is the most expensive benign condition in industrialised countries owing to the number of work days lost. LBP, by definition, includes pain below the costal margin but above the inferior gluteal folds. ${ }^{[1-3]}$ Definitions are given in Table 1. These include pain with and without nonneuropathic leg pain. Various studies have indicated that $80 \%$ of US adults experience LBP in their lifetime. ${ }^{[3,4]}$ In clinical practice, most practitioners have a superficial approach to this important clinical problem. Specific causes for LBP are uncommon and account for $<15 \%$ of all such cases. In $85 \%$ of cases LBP is nonspecific; it will improve within 6 weeks, irrespective of treatment.

The medical practitioner should try to establish whether the pain is mechanical or inflammatory. This distinction can often be made with the use of 'diagnostic red flags' (Table 2). Mechanical (nonspecific) LBP includes orthopaedic and neurosurgical causes, which may require surgical intervention. Epidemiological evidence suggests several risk factors for the development of LBP, including smoking, obesity and physically strenuous work. However, sedentary work and psychologically taxing work are also risk factors. Inflammatory back pain (IBP) is the hallmark symptom of spondyloarthritis. The features of IBP are listed in Table 3.

\section{Assessment of a patient with lower back pain}

There are multiple causes for LBP. It is important to note, however, that in a primary care setting the majority of patents have nonspecific LBP. Some important causes are outlined in Table 4.

The physical examination of the patient with LBP is important to distinguish benign from more serious causes of the pain. This includes inspection of the spine from multiple angles. Palpate along the spine for tenderness or abnormal 'steps', sometimes indicative of spondylolisthesis. The assessment of movement of the spine includes flexion, extension and lateral and rotational movement in two directions. A straight leg-raising test with the patient supine is deemed positive if pain is elicited between $10^{\circ}$ and $60^{\circ}$ and is indicative of compression at L4/L5. The Schober test is also important to assess the flexibility of the lumbar spine, which essentially measures stretch of the spine rather than the flexion angle (Fig. 1). The 'capsular pattern' of limitation at the lumbar spine is

\section{Table 1. Definitions}

- Acute lower back pain: an episode of lower back pain that resolves within 6 weeks

- Subacute lower back pain: pain persisting for 6 - 12 weeks

- Chronic lower back pain: persistent pain $>12$ weeks

- Nonspecific lower back pain: pain not attributed to recognisable or known pathology, e.g. infection, fracture

Table 2. Red flags in lower back pain

- Pain in patients $<20$ years and $>55$ years

- Pain not relieved on rest or posture modification

- Pain unchanged despite 2 - 4 weeks of treatment

- History of malignancy

- Immunosuppressed status

- Fever/malaise/weight loss

- High fracture risk, e.g. osteoporosis

- Neurological impairment

- Bladder or bowel dysfunction

- Severe morning stiffness as the primary complaint

- Inability to ambulate

Table 3. Features of inflammatory back pain

- Insidious onset

- Age $<45$ years

- Nocturnal pain

- Alternating buttock pain

- Pain with associated early morning stiffness lasting $>1$ hour

- Pain not relieved by rest

- Pain improving with exercise or activity

characterised by a limitation of movement in all planes, while at the neck flexion is usually not affected. This pattern of limitation of movement is generally associated with IBP. Mechanical causes of LBP will usually only limit movement in one plane. A discrepancy between the physical findings and anatomical or physiological principles is a way of identifying patients with major psychological factors. This should alert the clinician to the 'yellow flags' (Table 5). 
Table 4. Important causes of lower back pain

\begin{tabular}{|c|c|c|}
\hline Mechanical & Neurological & Systemic \\
\hline Disc herniation & Radiculopathy & $\begin{array}{l}\text { Inflammatory } \\
\text { spondyloarthropathy }\end{array}$ \\
\hline Spinal canal stenosis & Myelopathy & Metabolic bone diseases \\
\hline $\begin{array}{l}\text { Disc and segmental degradation, } \\
\text { e.g. facet arthropathy }\end{array}$ & Neuropathy & Neoplasia, including myeloma \\
\hline \multirow[t]{2}{*}{ Soft-tissue injuries } & Myopathy & $\begin{array}{l}\text { Infections of bone, disc or } \\
\text { epidura }\end{array}$ \\
\hline & Lumbosacral plexopathy & Referred pain \\
\hline
\end{tabular}
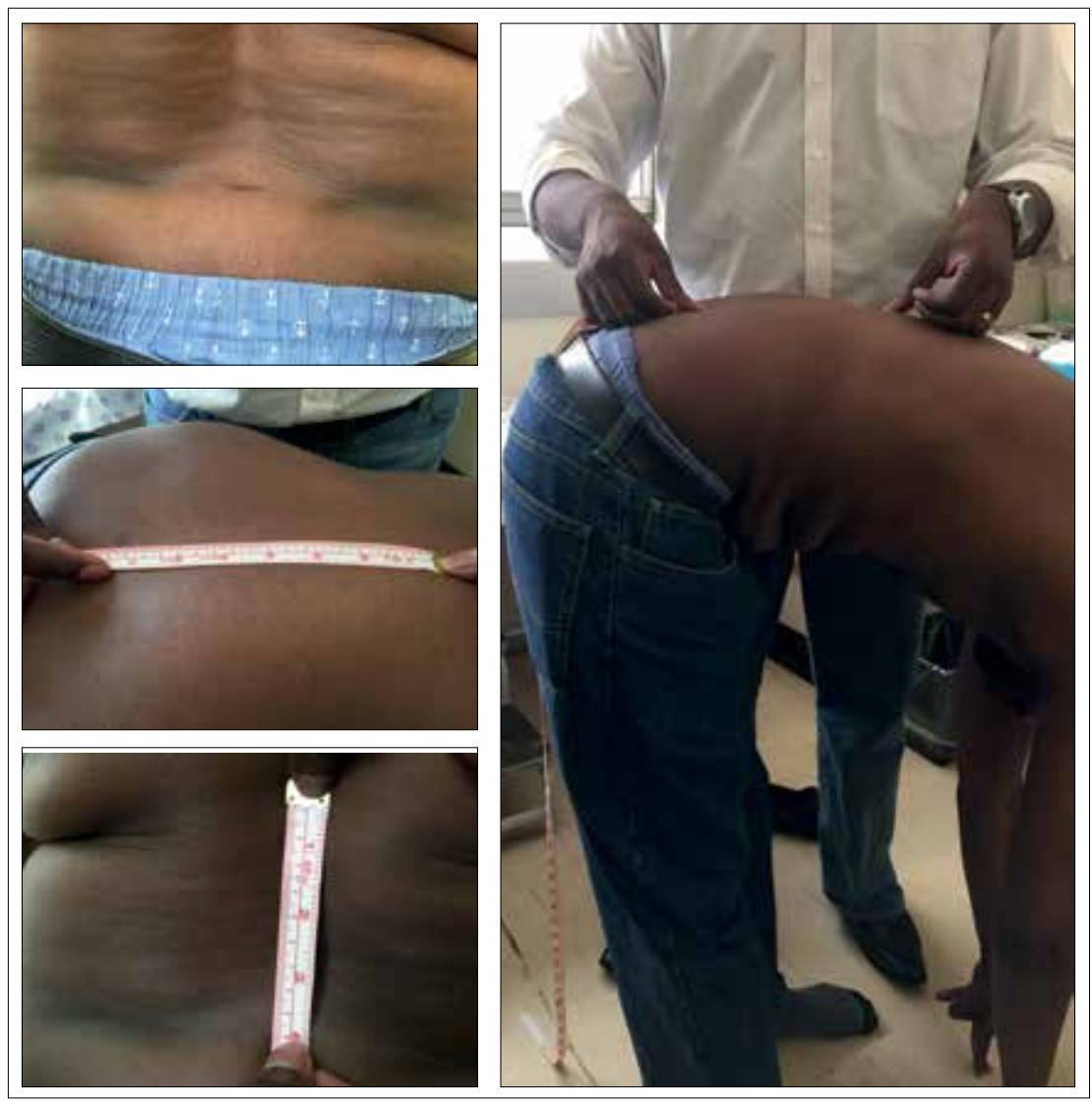

Fig. 1. The Schober test.

\section{Table 5. Yellow flags}

- Dissociation between verbal and non-verbal pain behaviour

- Compensable cause of injury

- Psychological or emotional factors, e.g. depression, anxiety, abusive relationships

- Narcotic drug requests

- Repeated failure of both medical and surgical therapy

- Disability or inability to return to work (unwillingness)

\section{Imaging}

Appropriate imaging of the spine is an essential part of the evaluation when a specific cause of LBP is suspected. Anteroposterior and lateral plain radiographs are helpful in assessing gross bone density, disc and vertebral body height and alignment. These should include a view of the pelvis. The characteristic radiographic feature of IBP is the presence of sacro-iliitis (Fig. 2). The classification criteria for ankylosing spondylitis (AS) are heavily weighted on the finding of sacro-iliitis on radiography. ${ }^{[5]}$ However, recent evidence has demonstrated that radiographs are not sensitive in the detection of early sacro-iliitis and newer criteria include other imaging modalities,

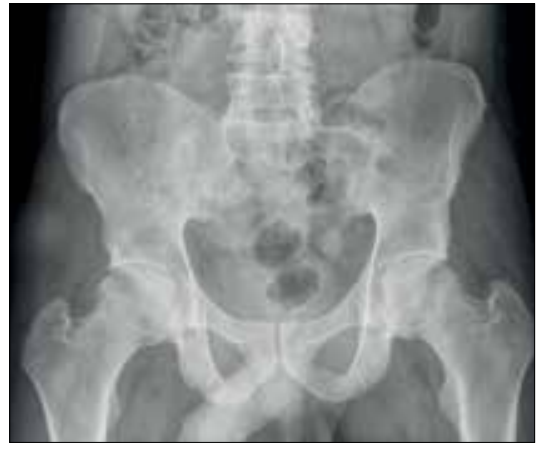

Fig. 2. Radiograph of patient with sacro-ilitis.

such as computed tomography (CT) scanning and magnetic resonance imaging (MRI), to diagnose IBP (non-radiographic inflammatory back pain). ${ }^{[6]}$ CT scanning is effective for assessing foraminal and extraforaminal nerve roots. It is thus most helpful if bony pathology is suspected. MRI is superior to CT for spinal imaging, but is more expensive. It is very effective for spinal cord or soft-tissue imaging, especially to diagnose sacro-iliitis. Other features of IBP include the presence of syndesmophytes, representing calcification of the spinal ligaments and culminating in the 'bamboo' spine, characteristic of longstanding AS. There may also be ankylosis of the posterior components of the vertebrae, and ultimately neglected patients with AS will develop a 'question mark posture'.

While the spondyloarthritides characteristically affect the spine, there are situations where the inflammation affects the entheses around peripheral joints rather than those around the spine (peripheral spondyloarthritis). ${ }^{[6]}$ This is often the case in reactive arthritis, where plantar fasciitis leads to development of a calcaneal spur, and Achilles tendinitis results in calcification of the Achilles tendon insertion (enthesis). Psoriatic arthritis usually affects the peripheral joints rather than the sacro-iliac joint. Spondyloarthritides, other than AS, often results in unilateral sacro-iliitis. Tuberculosis (TB) is an important cause of the latter included in the differential diagnosis. There is a strong relationship between the presence of HLA B27 and sacro-iliitis in patients with spondyloarthritis. ${ }^{[5,6]}$

\section{Approach to management}

Therapy or management of chronic LBP involves a multidisciplinary approach. The aim is to prevent a chronic vicious cycle by means of positive changes in the patient's beliefs, coping mechanism and physical ability. The team employs pharmacological and non-pharmacological strategies to realise the goal. 
Worldwide, the most commonly prescribed drugs for nonspecific LBP are the non-steroidal anti-inflammatory drugs (NSAIDs). There is evidence that they are more efficacious than placebo in the short term. ${ }^{[7]}$ Because of their side-effects, it is recommended that in chronic LBP they should be used for short periods during pain exacerbations only. The best available evidence recommends the use of both analgesia and an antidepressant in nonspecific (mechanical) chronic LBP. The most widely studied are the tricyclic antidepressants. They modulate pain sensation through blockage of the reuptake of neurotransmitters.

Non-pharmacological therapy includes information and reassurance. This is an essential component of therapy. The use of the biopsychosocial model to change a patient's beliefs around chronic LBP is important. Exercise therapy and avoidance of bed rest are also part of the management of LBP. Cognitive and behavioural therapy are helpful to change the patient's response to pain.

In the treatment of IBP the goal is to suppress the inflammation of the enthesis in an effort to prevent calcification, probably as a healing response after inflammation. Regular use of NSAIDs has been shown to prevent syndesmophyte formation. ${ }^{[8]}$ The current recommendation for the treatment of AS is that continuation of symptoms with persistent elevation of inflammatory markers after 3 months of NSAID use is an indication for specific targeted therapy against tumour necrosis factor (TNF). ${ }^{[9]}$ These agents are highly effective, but extremely expensive, making them unavailable for general use. Another disadvantage of this class of therapy is the risk of reactivating latent $\mathrm{TB}$.

\section{Summary}

Chronic LBP is a common condition, usually with a nonspecific cause. A diagnostic algorithm can be used to identify patients with non-mechanical back pain. The assessment should also include prognostic yellow flags to identify patients who are at risk. It is very important to differentiate IBP and to identify its cause. The management of such patients involves a multidisciplinary team approach, employing pharmacological and nonpharmacological strategies. IBP management has advanced considerably in recent years and usually requires the regular use of NSAIDs. Biological monoclonal antibodies directed against TNF should be used if NSAIDs have not resulted in improvement within 3 months and there is persistent elevation of inflammatory markers.

\section{References}

1. Deyo RA, Tsui-Wu YJ. Descriptive epidemiology of low-back pain and its related medical care in the United States. Spine (Phila Pa 1976) 1987;12:264.

2. Cassidy JD, Carroll LJ, Côté P. The Saskatchewan health and back pain survey. The prevalence of low back pain and related disability in Saskatchewan adults. Spine (Phila Pa 1976) 1998;23:1860.

3. Kelsey JL, White AA. Epidemiology of low back pain. Spine 1980;6:133-142.

4. Chou R, Qaseem A, Snow V, et al. Diagnosis and treatment of low back pain: A joint clinical practice guideline from the American College of Physicians and the American Pain Society. Ann Intern Med 2007;147:478.

5. Van der Linden S, Valkenburg HA, Cats A. Evaluation of diagnostic criteria for ankylosing spondylitis. A proposal for modification of the New York criteria. Arthritis Rheum 1984;27:361-368.

6. Rudwaleit M, van der Heijde D, Landewé R, et al. The development of Assessment of SpondyloArthritis International Society classification criteria for axial spondyloarthritis (part II): Validation and fin selection. Ann Rheum Dis 2009;68:777.

7. Rudwaleit $\mathrm{M}$, van der Heijde D, Landewé $\mathrm{R}$, et al. The Assessment of SpondyloArthritis International Society classification criteria for peripheral spondyloarthritis and for spondyloarthritis in general. Ann Rheum Dis 2011;70:25. 8. Wanders A, Heijde Dv, Landewé R, et al. Nonsteroidal antiinflammatory drugs reduce radiographic progression in patients with ankylosing spondylitis: A randomized clinical trial. Arthritis Rheum 2005;52:1756-1765.

9. Braun J, van den Berg R, Baraliakos X, et al. 2010 update of the ASAS/EULAR recommendations for the management of ankylosing spondylitis. Ann Rheum Dis 2011;70:896-904. [http://dx.doi. org/10.1136/ard.2011.151027] 